\title{
Comportement d'un massif renforcé par inclusions rigides verticales à inertie variable
}

\section{DIAS}

Université Joseph-Fourier LTHE, Grenoble, France

\section{J. GRIPPON}

Franki Fondation 11, rue du Dôme 69630 Chaponost
Le développement de réseaux routiers, autoroutiers, ou ferroviaires, ainsi que de grandes installations industrielles sur des sols de fondation de mauvaise qualité nécessite l'utilisation de techniques de renforcement visant à améliorer la performance globale de ces sols à la fois en termes de réduction de tassement et d'augmentation de la capacité portante. Parmi les différentes techniques disponibles, l'amélioration des sols de fondation en incorporant des inclusions rigides verticales semble être une solution particulièrement appropriée, car elle est facile à mettre en œuvre et ne nécessite pas de substitution d'importants volumes de sol compressible.

La technique consiste à réaliser un groupe d'inclusions régulièrement espacées à travers une couche de sol compressible jusqu'à un substratum sous-jacent de capacité portante plus importante. La charge de surface étant ainsi transférée vers le sol compressible par l'intermédiaire d'un matelas de transfert de charge réalisé sur ces inclusions. Les tassements différentiels à la base du matelas entre le sol compressible et les inclusions rigides conduisent à un " effet de voûte » en raison de mécanismes de cisaillement induits.

Une technique de fabrication d'inclusions rigides a été mise au point pour réaliser en une seule opération une inclusion verticale rigide de forme circulaire associée à une tête en forme de cône inversé.

Dans cet article, une étude numérique basée sur le cas concret d'un chantier à proximité de Bourgoin-Jallieu (France) est proposée afin de mettre en évidence le mode de fonctionnement de cette nouvelle technique dans le cas de dallages industriels.

Mots-clés : renforcement, inclusion rigide, modélisation numérique, dalettes, inertie variable.

\section{Behavior of a pile-supported embankment using rigid inclusions with variable inertia}

The increasing lack of good quality foundation soils allowing the development of roadway, motorway, or railway networks, as well as large scale industrial facilities, necessitates the use of reinforcement techniques aimed at improving the global performance of such soils, both in terms of settlement reduction and increasing load bearing capacity. Among the various available techniques, the improvement of foundation soils by incorporating vertical stiff inclusions appears to be a particularly 
appropriate solution, since it is easy to implement and does not require any substitution of significant volumes of soft soil.

The technique consists in driving a group of regularly spaced inclusions through the soft soil layer down to an underlying competent substratum, the surface load being thus transferred to this substratum by means of those reinforcing inclusions, which illustrates the case of a piled embankment. The differential settlements at the base of the embankment between the soft soil and the stiff inclusions lead to an "arching effect" in the embankment due to shearing mechanisms in the fill.

A technique for producing rigid inclusions has been developed to achieve in a single operation a rigid circular pile associated with a cone shaped head reversed on the place of a rigid circular pile with a distribution armed slab. In this article, a numerical study based on a real case (a pile-supported road near Bourgoin-Jallieu (France)) is proposed to highlight the functioning mode of this new technique in the case of industrial slabs.

Key words: improvement, vertical rigid pile, numerical modeling, pile cap, variable inertia.

\section{Introduction}

La réalisation de remblais renforcés par inclusions rigides verticales rigides pour supporter des charges réparties (route, culée de pont, remblai, sols industriels, stations d'épuration...) a fortement augmenté en France.

Le procédé traditionnel consiste en la réalisation d'une maille régulière d'inclusions rigides, combinée à un matelas qui permet le transfert de charge par cisaillement et qui peut aboutir à la création de voûtes de charge (Okyay, 2010 ; Low, 1999 ; Hewlett, 1988 ; Fioravante, 2011). Ces mécanismes dans le matelas conduisent à limiter les tassements absolus et à minimiser les tassements différentiels (Briançon, 2004 ; Simon, 2012 ; Jenck, 2005, Kempfert 1999 ; Ellis 2009a, 2009b ; Demerdash, 1996).
Afin d'avoir en partie inférieure de la plate-forme de transfert de charge une surface de contact plus importante permettant une meilleure répartition des efforts sur les inclusions, tout en maintenant un nombre d'inclusions identiques, une augmentation de la section de la tête de l'inclusion est possible.

Ce système existe sous la forme de dalettes de béton préfabriquées placées sur la tête de l'inclusion. L'inconvénient majeur de ce dispositif réside dans le fait qu'il nécessite deux interventions distinctes (achèvement de l'inclusion et installation de la dallette de béton préfabriquée) et est difficile à mettre en place.

La notion d'inclusions rigides à inertie variable (INIV) permet donc de réaliser une inclusion circulaire associée à une tête conique. Elle peut être réalisée en une seule opération (Fig. 1) et assurer ainsi la continuité de l'inclusion.

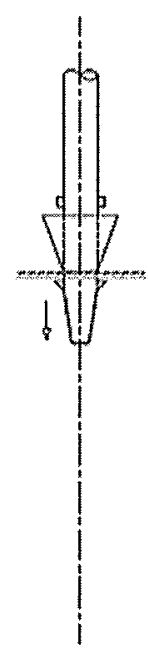

Mise en fiche

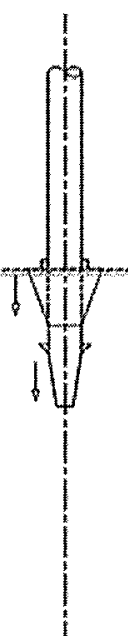

Verrouillage et fonçage

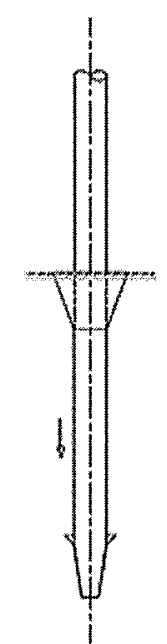

Déverrouillage et fonçage

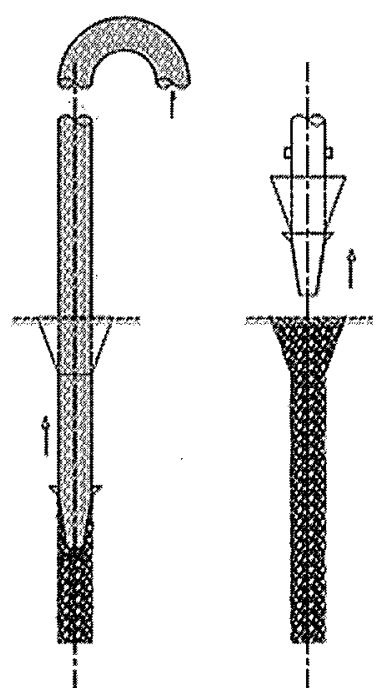

Bétonnage Inclusion terminêe 
Description du projet

2.8

\section{Informations générales}

Dans le cadre de la création d'un regroupement de plusieurs bâtiments hospitaliers sur un même site au sein de la ville de Bourgoin-Jallieu (France), le gouvernement français a décidé de créer une route $2 \times 2$ voies en élargissant la route existante et de réaliser un carrefour d'accès à ce Médipole. Ces ouvrages ont été réalisés par la mise en place de remblais sur des sols compressibles.

Les travaux d'amélioration par inclusions rigides ont été effectués sur une longueur d'environ $400 \mathrm{~m}$. La solution initiale consistait en la création d'inclusions rigides associées à des dallettes de béton préfabriqué. Une autre solution innovante a été proposée : la création d'inclusions rigides circulaires d'un diamètre de $0,35 \mathrm{~m}$ avec un diamètre de la tête élargie de $0,80 \mathrm{~m}$ (inclusions rigides à inertie variable) selon une maille régulière pour renforcer des sols fortement compressibles.

Les inclusions rigides à inertie variable ont été associées à un renforcement géosynthétique horizontal avant la réalisation des remblais et la création de la chaussée. Le choix des inclusions rigides à inertie variable a été fait pour plusieurs raisons : sa réalisation facile (pas besoin de mettre en œuvre des dallettes de béton préfabriqué), la réduction du temps d'exécution, l'augmentation de l'efficacité et des économies importantes en termes de matériaux (béton et acier).

\section{9}

\section{Contexte géotechnique}

Le lieu de réalisation de l'ouvrage renforcé (Fig. 2) est un ancien marécage plus ou moins sec avec une nappe phréatique à faible profondeur. Les différentes investigations géotechniques ont mis en évidence la succession lithologique suivante :

- une couverture de limon argileux brun à noirâtre ;

- des alluvions récentes composées de trois ensembles principaux relativement hétérogènes :

- principalement de l'argile alluviale, plastique, gris à beige, compressible à très compressible,

- tourbe brune à noire à base de plantes décomposées,

- alluvions argilo-limoneux humide et très compressibles,

- une couche de gravier sableux compacté. L'horizon rigide est situé à environ 5 mètres de profondeur.

\section{Modélisation numérique}

L'étude numérique proposée vise à mieux comprendre le comportement des plates-formes de transfert de charge réalisées sur inclusions rigides à inertie variable. Cette étude académique utilise la géométrie réelle et les propriétés des matériaux constitutifs du site de Bourgoin-Jallieu.

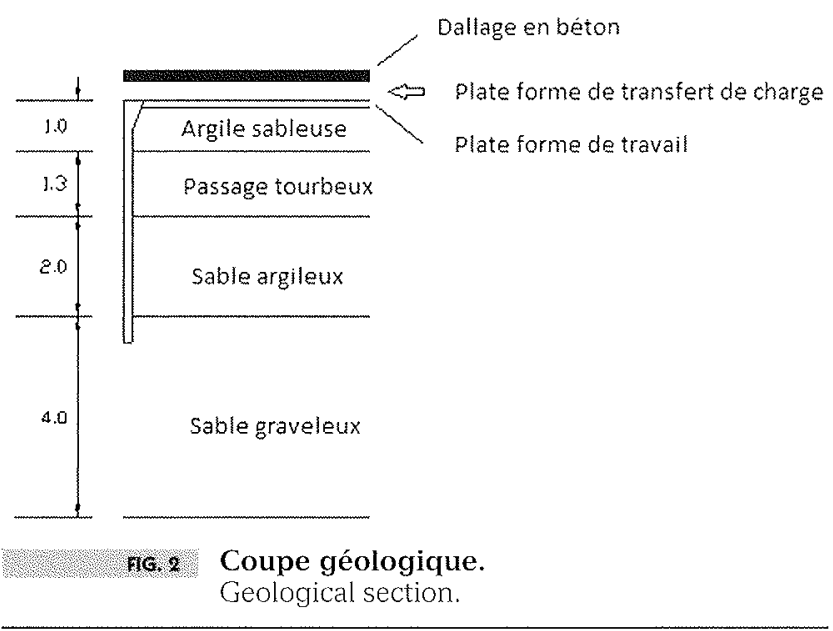

Une cellule élémentaire représentative d'un motif de maille carrée de 2,5 m de côté a été considérée. La figure 3a représente la vue latérale de l'inclusion et la $3 \mathrm{~b}$ la cellule unitaire simulée dans le modèle numérique. La géométrie 3D est simplifiée à une géométrie bidimensionnelle en configuration axisymétrique en utilisant un rayon équivalent de $1,41 \mathrm{~m}$ pour la cellule élémentaire. Un modèle numérique par éléments finis a été défini à l'aide du code de calcul Plaxis. Ce type de modélisation (simplification 3D vers le 2D) conduit à des résultats avec une bonne précision par rapport à une modélisation 3D. L'erreur entre les modèles axisymétriques et 3D est inférieur à $9 \%$ (Nunez, 2007).

\section{-3.}

\section{Modèles numériques}

La géométrie étudiée (Fig. 3b) est constituée de 4,3 m de hauteur de couches horizontales compressibles supposées homogènes (argile sableuse, tourbe et de sable argileux). Ces horizons sont renforcés par des inclusions rigides verticales circulaires de $0,35 \mathrm{~m}$ de diamètre. Les inclusions ont $4,8 \mathrm{~m}$ de longueur et sont espacées de $2,5 \mathrm{~m}$. Sur l'argile sableuse, une plateforme de travail de $0,3 \mathrm{~m}$ de hauteur a été créée. La plate-forme de $0,5 \mathrm{~m}$ de hauteur a été réalisée sur les inclusions. La figure $3 a$ présente le détail de la tête de l'inclusion à inertie variable. Le cas de référence de cette étude a été dimensionné pour tenir compte de la présence d'une structure à la surface. Une dalle de béton d'une épaisseur de 0,22 m est placée sur la plateforme de transfert de charge. Une surcharge de $25 \mathrm{kPa}$ est ensuite appliquée sur cette structure.

Une campagne d'essais géotechniques (cedomètres, essais triaxiaux, un essai de chargement et des pénétromètres) ont permis d'obtenir les caractéristicues géomécaniques des couches de sol. Une surconsolidation de $10 \mathrm{kPa}$ a été initialement appliquée à la couche d'argile sableuse.

Le modèle de comportement adopté pour les couches compressibles est le modèle Soft Soil mis en œuvre dans Plaxis. Le tableau I présente les paramètres géomécaniques retenus pour ces couches.

Pour les autres couches de sol (plate-forme de transfert de charge, plate-forme de travail et l'horizon de gravier sableux), un modèle de comportement élastique linéaire parfaitement plastique avec un cri- 

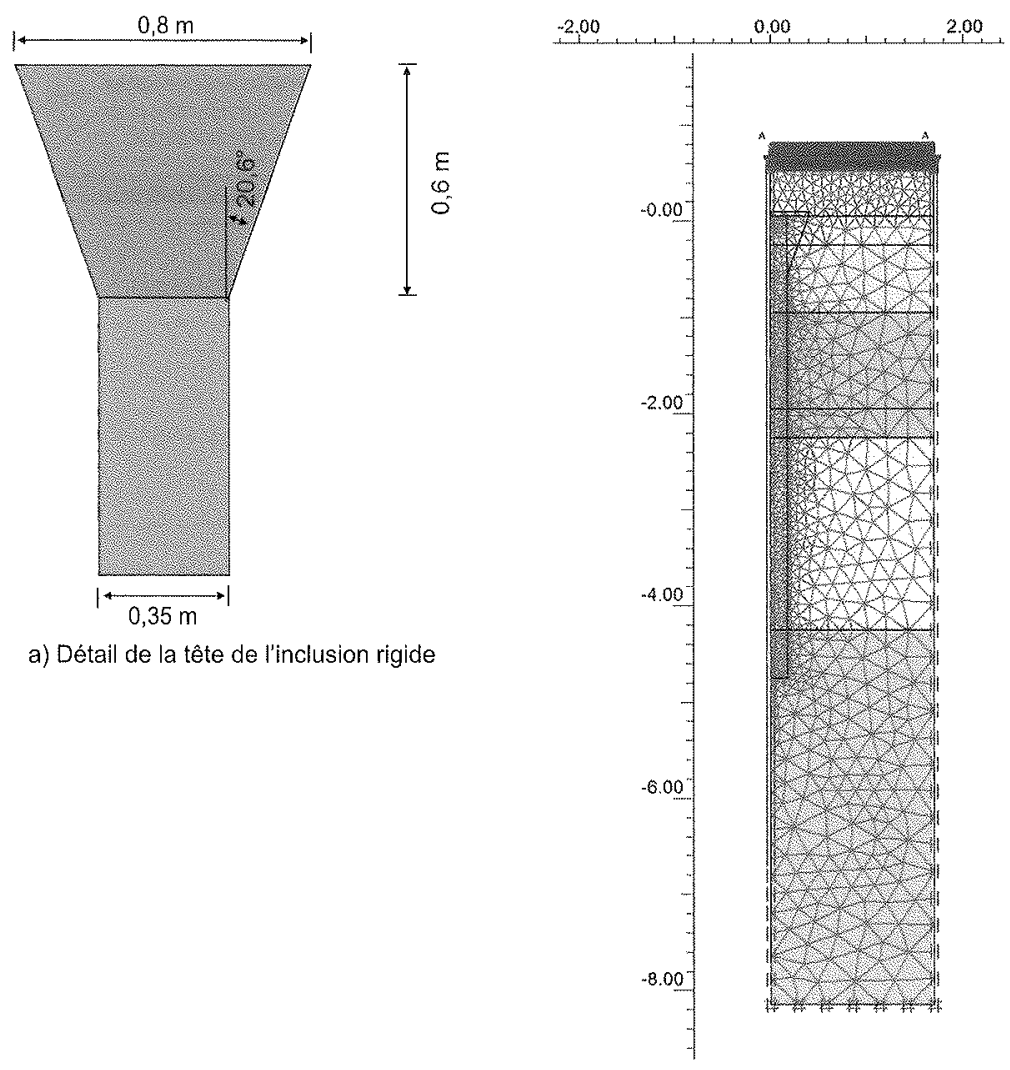

b) Modèle numérique adopté

n6 3 Détail de la cellule élémentaire simulée. Detail of the unit cell modelled.

\begin{tabular}{|c|c|c|c|c|c|c|}
\hline TABIEAUI I & \multicolumn{6}{|c|}{$\begin{array}{l}\text { Paramètres géomécaniques pour la } \\
\text { simulation des sols en utilisant le modèle } \\
\text { Soft Soil. } \\
\text { Parameters for the geomechanical simulation } \\
\text { using the Soft Soil constitutive model. }\end{array}$} \\
\hline hrontwor & & 1): & (2) & e. & $f(1)$ & Y: (I. Nm) \\
\hline \multicolumn{2}{|l|}{ Argile sableuse } & 0,45 & 0,04 & 1,17 & 29,4 & 15 \\
\hline \multicolumn{2}{|l|}{ Tourbe } & 1,31 & 0,13 & 2,46 & 19,3 & 15 \\
\hline \multicolumn{2}{|c|}{ Sable argileux } & 0,45 & 0,04 & 1,17 & 23,2 & 18 \\
\hline
\end{tabular}

tère de rupture de type Mohr-Coulomb a été adopté (tableau II). Pour les éléments de structure, de l'élasticité linéaire a été utilisée.

Tous les calculs numériques ont été réalisés en conditions drainées.

Des interfaces frottantes ont été placées entre les inclusions et les couches de sol compressible. Une contrainte limite de frottement $q \mathrm{~s}=30 \mathrm{kPa}$ a été utilisée.

Les calculs numériques ont été analysés en efficacité en termes de contrainte. Différents auteurs proposent ce terme, afin de déterminer l'efficacité du renfort (Terzaghi, 1943 ; Hewlett et Randolph, 1988 ; British Standard BS8006, 1995 ; Guido et al., 1987 ; Russell et Pierpoint, 1997 ; Rogbeck et al., 1998). L'efficacité en termes de contraintes représente le rapport entre la charge transmise à la tête de l'in- clusion et la charge totale sur la maille élémentaire. Un autre paramètre a été défini l'efficacité en termes de tassement qui représente la réduction en termes de tassement calculé par le rapport entre le tassement du dallage renforcé par inclusions rigides par rapport au tassement du dallage en champ libre. La définition de ce ratio est définie par la formule suivante :

$$
E_{\text {Tassement }}=1 \frac{S_{\text {renforcé }}}{S_{\text {nonrenforcé }}}
$$

où $S_{\text {renforcé }}$ représente le tassement du dallage renforcé par inclusions rigides et $S_{\text {non renforcé }}$ le tassement du dallage en champ libre.

\section{4 \\ Résultats}

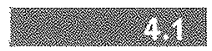

\section{Cas de référence}

La figure 4 présente le champ de déplacement vertical dans le massif de sol, le tassement maximum est situé à l'interface entre la plate-forme de travail et le matelas de transfert de charge entre les deux inclusions. La figure 5 permet d'identifier clairement le point neutre où le tassement du sol de fondation et celui de l'inclusion sont égaux et où l'inclusion atteint son chargement maximal. A ce stade, la contrainte maximale verticale dans l'inclusion est égale à $2490 \mathrm{kN} / \mathrm{m}^{2}$. 


\begin{tabular}{|c|c|c|c|c|c|c|c|}
\hline \multirow[t]{2}{*}{ rabisku II } & \multicolumn{7}{|c|}{$\begin{array}{l}\text { Paramètres géomécaniques pour les autres couches de sol et les éléments structurels } \\
\text { Geomechanical parameters of other soil layers and structural elements. }\end{array}$} \\
\hline & SolMayer & E IMPa & vis & $\phi(?)$ & e $(k) a)$ & Y. $1:$ & 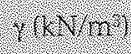 \\
\hline \multicolumn{2}{|c|}{ Plate-forme de transfer de charge } & 50 & 0,3 & 35 & 0 & 5 & 20 \\
\hline \multicolumn{2}{|c|}{ Plate-forme de travail } & 30 & 0,3 & 30 & 0 & 0 & 19 \\
\hline \multicolumn{2}{|c|}{ Gravier sableux } & 90 & 0,3 & 41 & 10 & 5 & 19 \\
\hline \multicolumn{2}{|c|}{ Dallage en béton } & 11000 & 0,2 & & & & 25 \\
\hline \multicolumn{2}{|c|}{ Inclusions rigides } & 8000 & 0,2 & & & & 25 \\
\hline
\end{tabular}

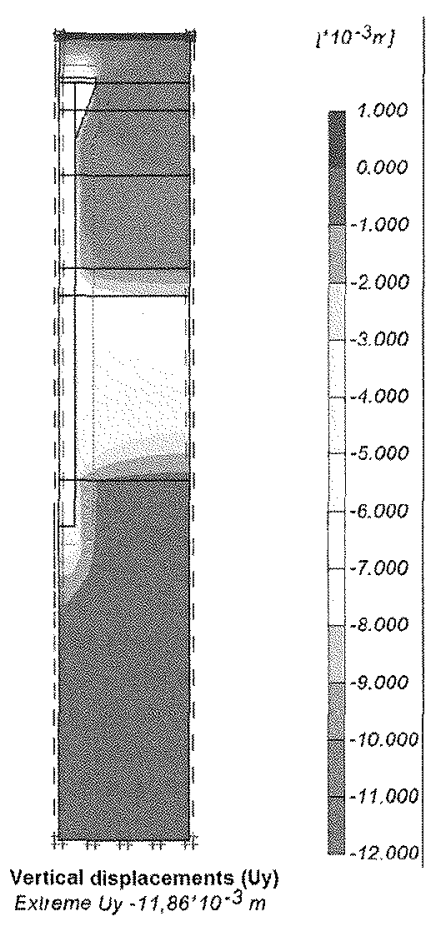

F6. 4 Champ de déplacement vertical dans le massif.

Vertical displacement field in the soil mass.
Dans le cas de référence, le moment de flexion dans l'inclusion atteint la valeur de $14,08 \mathrm{kN} . \mathrm{m} / \mathrm{m}$.

Un point intéressant est la répartition du frottement le long du fût de l'inclusion. Dans le cas d'un renforcement INIV, le frottement est seulement saturé à la pointe de l'inclusion (frottement ultime atteint). Cela est dû à l'inclinaison de la tête de l'inclusion qui ne permet pas l'augmentation du frottement le long de l'inclusion sur les 0,6 m du haut de la tête. Dans cette zone, le frottement négatif ne peut se développer et est donc transféré vers les couches inférieures. Dans le cas de ce type d'inclusions, un soin tout particulier doit être porté sur les conditions de frottement en pointe d'inclusion. En termes de chargement dans la zone renforcée, du fait de la faible hauteur entre la tête de l'inclusion et le dallage, des cisaillements partiels se développent mais ne peuvent conduire à la formation d'un voute complète. Une concentration des contraintes se produit donc sur la tête d'inclusion.

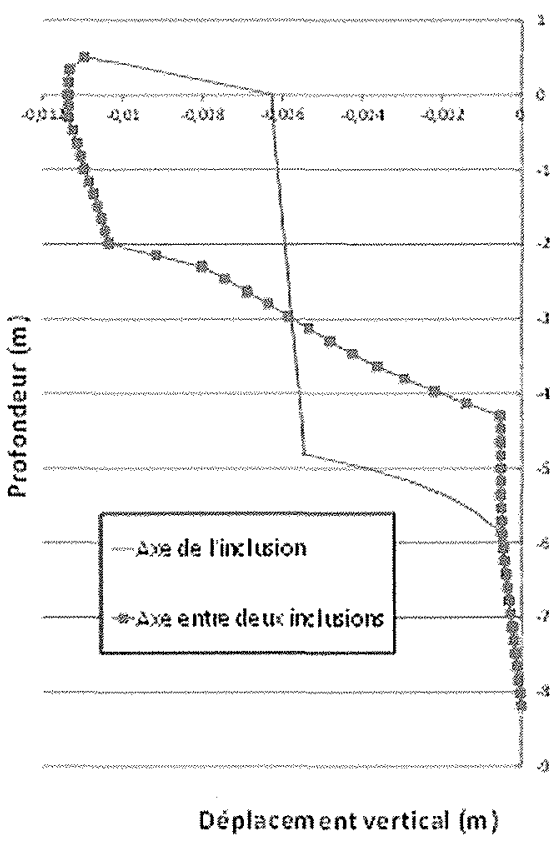

no. 5 Déplacement vertical de l'inclusion et entre deux inclusions.

Vertical displacement of the inclusion and between two inclusions.
En raison de l'inclinaison de la tête de l'inclusion, une traction de $0,5 \mathrm{MPa}$ apparaît à l'angle où l'inertie de l'inclusion devient variable. Le béton constituant l'inclusion peut toutefois reprendre une contrainte de traction de 1,2 $\mathrm{MPa}$

\section{(3)}

\section{Influence de l'épaisseur du matelas}

L'étude de l'influence de l'épaisseur du matelas permet de mettre en évidence l'épaisseur nécessaire pour développer une voûte complète au-dessus des inclusions. On peut voir que, pour une valeur supérieure à 0,70 $\mathrm{m}$ pour l'épaisseur de matelas (cas de référence), une diminution du moment de flexion est observée. Cet effort atteint une valeur négligeable pour une épaisseur de matelas égale à la distance entre les inclusions verticales. Il n'est donc pas nécessaire d'augmen- 


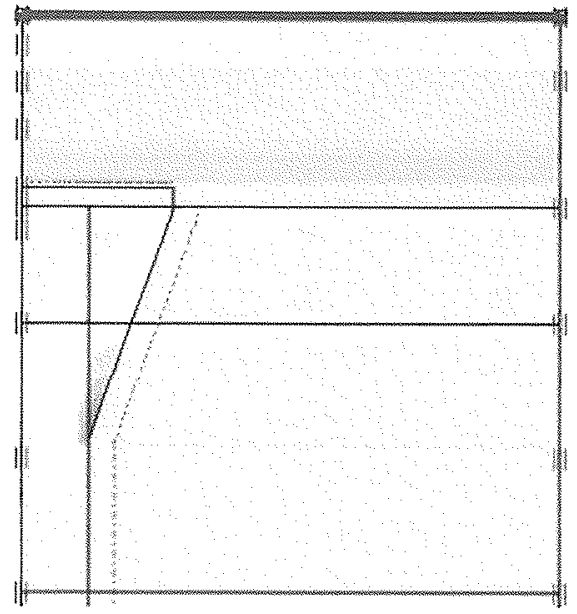

16.6. Contrainte de cisaillement.

Shear stress.

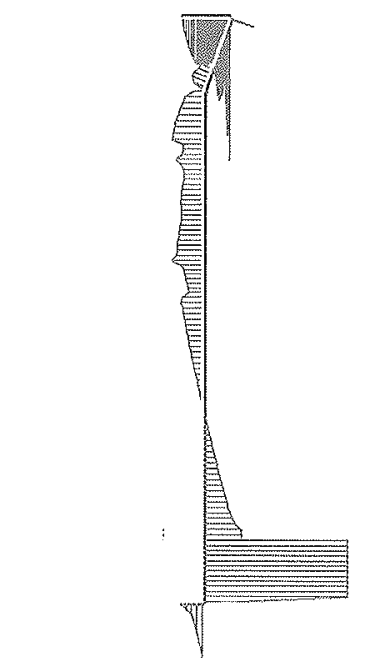

M. 1
Frottement le long de l'inclusion (maximum $110 \mathrm{kPa}$ ).

Friction along the inclusion (maximum $110 \mathrm{kPa}$ ).

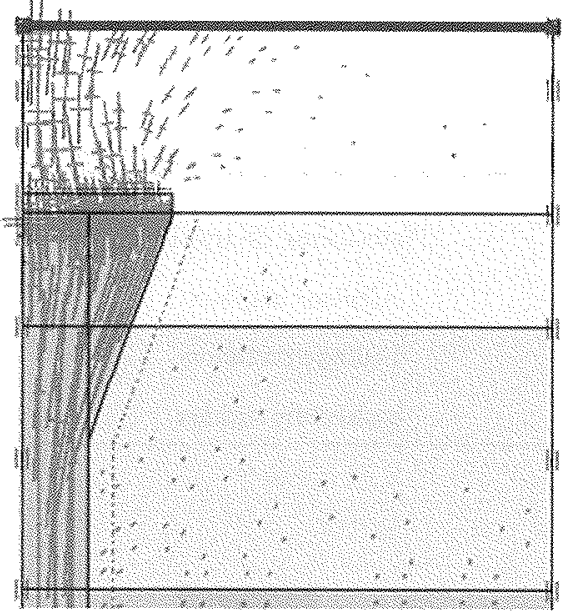

FiG.

Contraintes principales (compression maximale de 2,21 MPa).

Principal stress (maximum compression of 2.21 MPa).

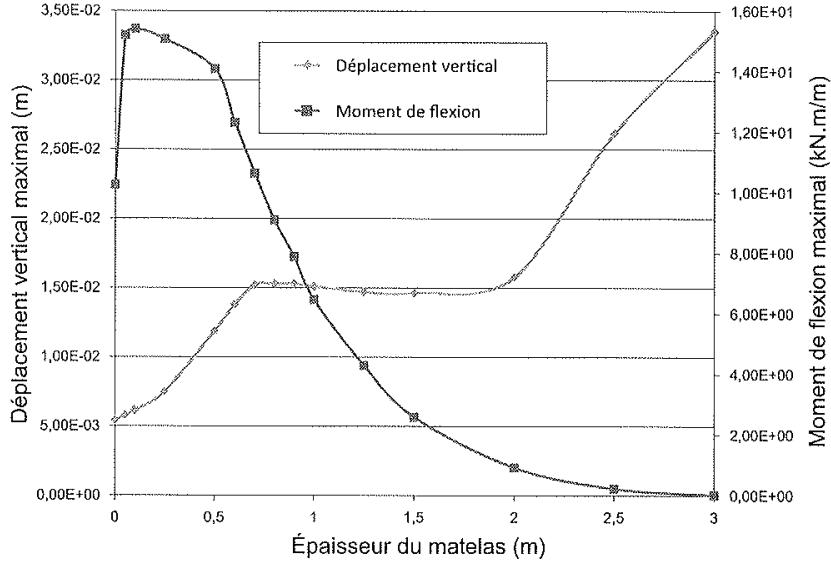

R6. 9 Déplacement vertical maximal et moment de flexion maximal suivant l'épaisseur du matelas.

Maximum vertical displacement and maximum bending moment according to the thickness of the mattress.

ter l'épaisseur du matelas à la valeur de la maille pour permettre le développement de l'effet de voûte.

\section{4.}

\section{Influence du diamètre de l'inclusion rigide}

Pour montrer l'influence d'une tête à inertie variable, une étude préliminaire prenant en compte une inclusion circulaire conventionnelle a été mise en œuvre. L'influence du diamètre de l'inclusion a été étudiée par une étude paramétrique basée sur le cas de référence. Le diamètre de l'inclusion a été modifié dans un intervalle allant de $0,35 \mathrm{~m}$ à $2 \mathrm{~m}$.
La figure 9 montre l'évolution du déplacement maximal en fonction de l'augmentation du diamètre de l'inclusion. La diminution de ce paramètre est importante jusqu'à un diamètre de $0,50 \mathrm{~m}$. Après cette valeur du diamètre, le déplacement vertical atteint un seuil. En termes de moment de flexion dans la dalle, cette valeur du diamètre de l'inclusion est une zone intéressante, où l'évolution du moment de tension change. Avant cette valeur, on observe une augmentation jusqu'à un maximum de $13 \mathrm{kN} . \mathrm{m} / \mathrm{m}$, puis on observe une diminution du moment de flexion.

Dans cette configuration, une inclusion de diamètre $0,7 \mathrm{~m}$ correspond au cas où le dallage est le plus chargé et où sa contribution à la limitation du déplacement vertical est la plus élevée. Pour des valeurs plus élevées

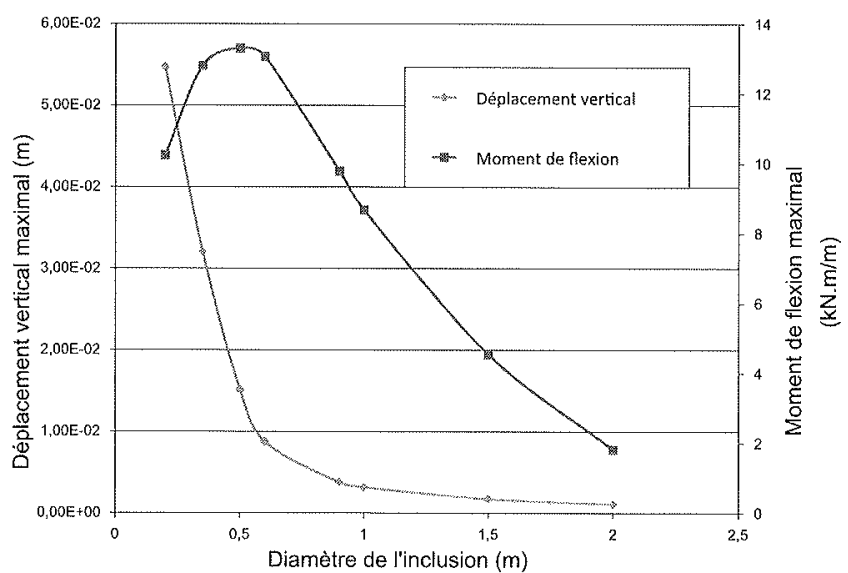

FG 10 Déplacement vertical maximum dans le sol et moment de flexion dans le dallage en fonction du diamètre en tête d'inclusion.

Maximum vertical displacement in the soil and bending moment in the pavement according to the diameter at the top of inclusion. 
du diamètre de l'inclusion, la flexion du dallage est moins prononcée à cause du diamètre de l'inclusion et conduit ainsi à une diminution du moment de flexion. Ces résultats dépendent de la hauteur du matelas (qui était une constante lors de l'étude paramétrique proposée ici).

La figure 11 présente les efficacités en termes de contraintes et de tassement. Ces deux quantités présentent la même évolution. Leur valeur augmente avec le diamètre de l'inclusion.

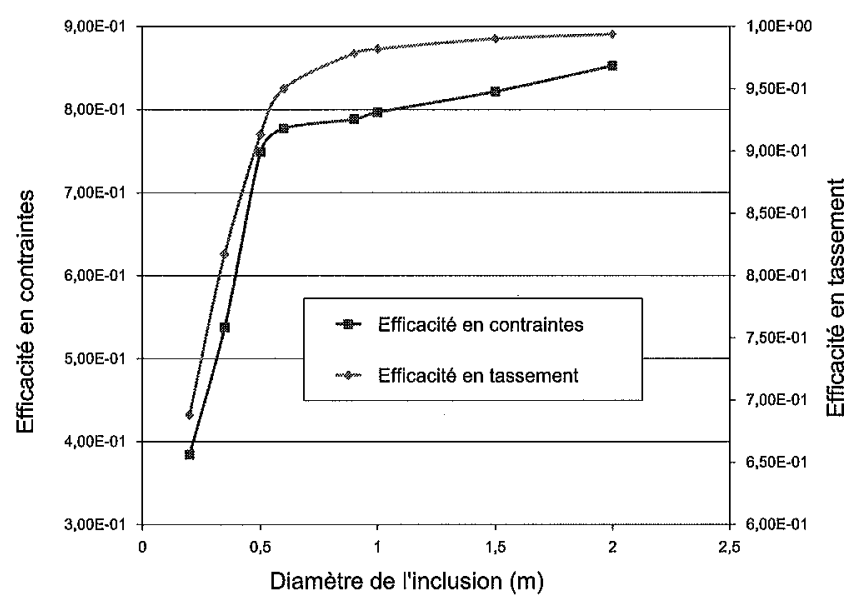

116. 11 Efficacité en termes de contraintes et de tassement.

Efficacy in terms of stress and settlement.

\section{Comparaison avec une inclusion à inertie variable (inclusions INIV)}

Pour comparer le comportement des inclusions circulaires avec celles qui ont une inertie variable, une étude paramétrique sur la tête de l'inclusion de diamètre a été entreprise. Le diamètre de la partie inférieure de l'inclusion a été fixé à $0,35 \mathrm{~m}$, puis le diamètre de la tête a été modifié dans une plage allant de $0,35 \mathrm{~m}$ à $2 \mathrm{~m}$.

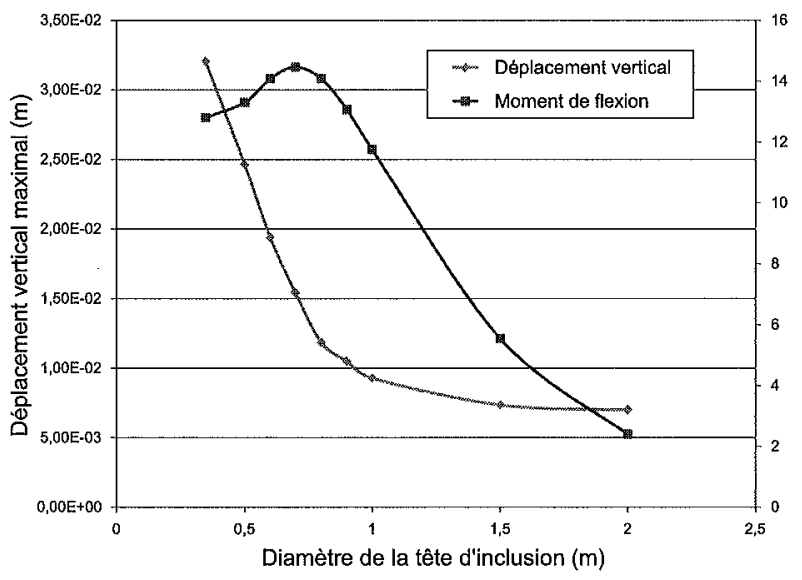

Déplacement vertical maximum dans le sol et moment de flexion dans le dallage en fonction du diamètre en tête d'inclusion. Maximum vertical displacement in the soil and bending moment in the pavement according to the diameter at the top of inclusion.

Le comportement de l'inclusion rigide à inertie variable est très proche du comportement de l'inclusion circulaire conventionnelle. La figure 12 présente une diminution du déplacement verticale jusqu'à un plateau avec l'augmentation du diamètre de la tête d'inclusion. Dans un même temps pour le moment de flexion on observe une augmentation puis une diminution du moment de flexion avec un passage par un pic pour une valeur du diamètre de la tête d'inclusion de $0,7 \mathrm{~m}$.

Dans la suite de l'étude, les comparaisons entre les deux types d'inclusions seront toujours effectuées en considérant le diamètre de la tête.

La figure 13 montre la comparaison entre les deux types d'inclusions en termes de déplacements verticaux. Il est intéressant de noter que pour un même diamètre de la tête, les inclusions circulaires sont plus efficaces et permettent de mieux réduire les déplacements à l'interface entre la plate-forme de travail et le matelas de transfert de charge.

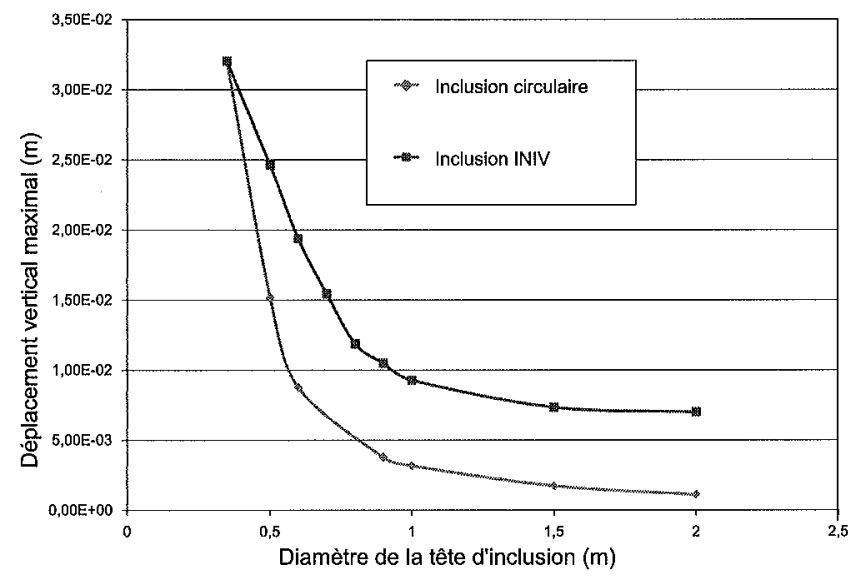

.4. vertical maximal entre les inclusions circulaires et celles à inertie variable.

Confrontation in terms of maximum vertical displacement between circular inclusions and variable inertia ones.

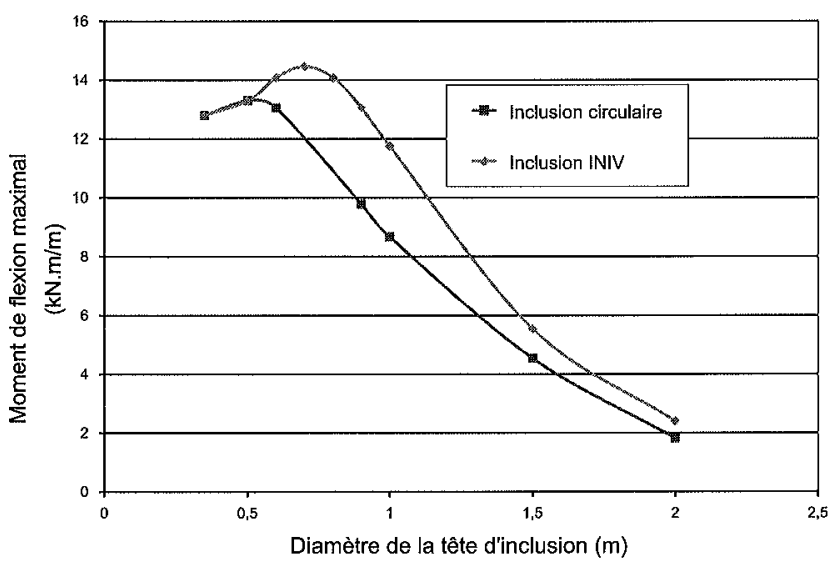

76. 14. Confrontation en termes de moment de flexion entre les inclusions circulaires et celles à inertie variable.

Comparison in terms of bending moment between the circular inclusions and the variable inertia ones. 


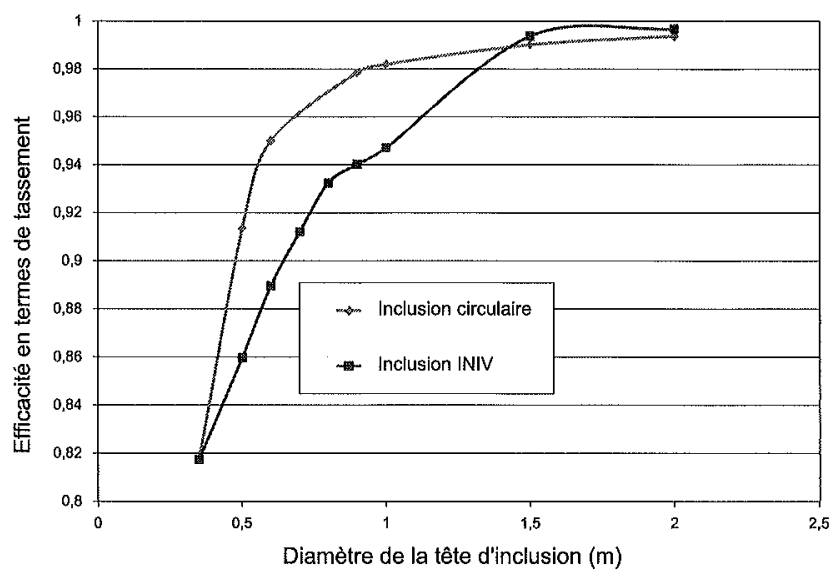

76. 15. Efficacité en termes de tassement pour les inclusions circulaires et celles à inertie variable.

Efficacy in terms of settlement for circular and variable inertia inclusions.

La même tendance est observée pour les moments de flexion. Les inclusions circulaires sont plus efficaces. Ce qui est dû au déplacement relatif entre le sol et la tête de l'inclusion qui est plus élevé dans le cas de l'inclusion à inertie variable. Toutefois, le volume de matériau incorporé (tableau III) est significativement plus faible dans le cas d'une inclusion rigide à inertie variable par rapport à une inclusion circulaire de même diamètre de tête $(76 \%$ de réduction sur une tête de diamètre $0,8 \mathrm{~m}$ ).

Si l'on compare une inclusion à inertie variable (diamètre de la tête de $0,80 \mathrm{~m}$ et fût de $0,35 \mathrm{~m}$ ) et une inclusion circulaire de diamètre $0,35 \mathrm{~m}$, la réduction du déplacement vertical est beaucoup plus important (baisse de 63,2 \%) dans le premier cas. Toutefois, le moment de flexion induit dans le dallage est plus important dans le cas des inclusions à inertie variable (hausse de 7,4\%).

\section{5 \\ Conclusion}

Le mode de fonctionnement des inclusions rigides à inertie variable est différent de celui des inclusions circulaires conventionnelles. Le frottement négatif ne peut pas être totalement développé au niveau de la tête de l'inclusion et les efforts sont donc redistribués le long du fût de l'inclusion. Une plus grande attention doit être portée quant aux conditions de frottement à la pointe de l'inclusion.

La comparaison entre les inclusions à inertie variable avec les circulaires a montré que les deux types d'inclusions ont le même comportement si l'on considère les moments de flexion dans le dallage ou l'efficacité en termes de contraintes ou de tassement.

Avec le même diamètre de la tête, l'inclusion circulaire permet une réduction plus importante du tassement et du moment de flexion induit dans le dallage que l'inclusion à inertie variable. Toutefois, le volume nécessaire de matériau devant être inclus est beaucoup plus important pour une inclusion circulaire de même diamètre.

En comparant une inclusion à inertie variable dans le cas de référence et une inclusion circulaire de même diamètre de fût, la diminution du tassement vertical est plus importante dans le cas des inclusions à inertie variable. Toutefois, le moment induit dans le dallage en incluant les inclusions à inertie variable reste plus grand.

L'élargissement du diamètre de la tête à une valeur de $1,00 \mathrm{~m}$ pour une inclusion à inertie variable de fût de diamètre $0,35 \mathrm{~m}$ dans la configuration de référence permet aux inclusions à inertie variable d'être plus efficaces que les inclusions circulaires de diamètre de fût identique en termes de réduction de tassement vertical et également en termes de moment de flexion dans le dallage.

Volume nécessaire de béton pour les différents types d'inclusions. Necessary concrete volume for the different types of inclusions.

\begin{tabular}{|c|c|c|c|c|c|c|c|c|}
\hline Diamètre de la tête d'inclusion (m) & 0,35 & 0,50 & 0,60 & 0,70 & 0,80 & 0,90 & 1,00 & 1,50 \\
\hline Volume de l'inclusion circulaire $\left(\mathrm{m}^{3}\right)$ & 0,46 & 0,94 & 1,36 & 1,85 & 2,41 & 3,05 & 3,77 & 8,48 \\
\hline Volume de l'inclusion à inertie variable $\left(\mathrm{m}^{3}\right)$ & 0,46 & 0,49 & 0,51 & 0,54 & 0,57 & 0,60 & 0,64 & 0,86 \\
\hline
\end{tabular}


Briançon L., Kastner R., Simon B., Dias D. - État des connaissances. Amélioration des sols par inclusions rigides. Actes du colloque international sur l'amélioration des sols en place (Dhouib A., Magnan J.-P., Mestat P. Eds.), Paris, Presses des Ponts et Chaussées, 2004.

BS8006, British Standards - Code of Practice for Strengthened/Reinforced Soils and Other Fills. Section 8 : Design of Embankments with Reinforced Soil Foundation on Poor Ground. British Standard Institution, London, 1995, p. 80-121.

Demerdash M.A. - An experimental study of piled embankments incorporating geosynthetic basal reinforcement. Thèse de doctorat, University of Newcastleupon-Tyne, 1996

Ellis E.A., Aslam R. - Arching in piled embankments : comparison of centrifuge tests and predictive methods. Part 1 of 2, Ground Engineering, June 2009a, p. 34-38.

Ellis E.A., Aslam R. - Arching in piled embankments : comparison of centrifuge tests and predictive methods - Part 2 of 2, Ground Engineering, July 2009b, p. 28-31.
Fioravante V. - Load transfer from a raft to a pile with an interposed layer. Géotechnique, 61, n² 2, 2011, p. 121-132.

Guido V.A., Kneuppel J.D., Sweeny M.A. Plate loading tests on geogridreinforced earth slabs. Proceedings of the Geosynthetics '87, New Orleans, USA, IFAI, 1987, p. 216-222.

Hewlett W.J., Randolph M.F. - Analysis of piled embankments. Ground Engineering, 21 (3), 1988, p. 12-18.

Jenck O., Dias D., Kastner R. - Soft Ground improvement by vertical rigid pilesTwo-dimensional physical modelling and comparison with current design methods. Soils and Foundation, vol. 45, $n^{\circ} 6$, 2005, p. 15-31.

Kempfert H.G., Zaeske D., Alexiew D. Interactions in reinforced bearing layers over partially supported underground. Proc. of the 12th European Conf. on Soil Mechanics and Geotechnical Engineering, 7-10 june 1999, Amsterdam, PaysBas. Rotterdam. Balkema, 1999, vol. 3, p. 1527-1532.

Low B.K., Tang S.K., Choa V. - Arching in piled embankments. Journal of Geotechnical and Geoenvironmental Engineering, vol. $120, n^{\circ} 11,1994$, p. 1917-1938.
Nunez M.A., Dias D., Poilpré, C., Kastner, $R$. - Influence of vertical rigid inclusions as ground improvement technique over a roadway embankment. 2D and 3D numerical modelling. 5th International Workshop on Application of Computational Methods in Geotechnical Engineering, Guimaraes, Portugal, 2007.

Okyay U.S. - Étude expérimentale et numérique des transferts de charge dans un massif renforcé par inclusions rigides. Application à des cas de chargements statiques et dynamiques. $\mathrm{PhD}$ thesis, université de Lyon, INSA, Lyon, 2010.

Russell D., Pierpoint N. - An assessment of design methods for piled embank ments. Ground Engineering, 30 (11), 1997, p. 39-44.

Rogbeck Y., Gustavson S., Södergren I Lindquist D. - Reinforced pile embankments in Sweden - design aspects. Proceedings 6th Int. Conf. on Geosynthetics, Atlanta, vol. II, 1998, p. 755-762.

Simon B. - Amélioration des sols par inclu sions rigides. Presses des Ponts, 2012.

Terzaghi K. - Theoretical soil mechanics. J. Wiley and Sons, New York, 1943 